\title{
Inteligencia artificial: Desarrollo de algoritmos de clasificación y segmentación en radiografía de tórax
}

\author{
Enzo Raschio $A^{{ }^{*}}$, Cassandra Contreras $\boldsymbol{R}^{2}$, Felipe Allende $\mathbf{N}^{1}$, Pablo Maturana $\mathbf{Q}^{1}$.
}

1. Tecnólogo Médico con mención en Radiología y Física Médica. Universidad Mayor, Santiago, Chile.

2. Tecnóloga Médica con mención en Radiología y Física Médica. Universidad Mayor, Santiago, Chile.

\section{Artificial Intelligence: Development of classification and segmentation algorithms in chest radiography}

\begin{abstract}
Resumen
Los algoritmos de inteligencia artificial han presentado un gran avance en las tareas relacionadas al reconocimiento de imágenes, siendo capaces de identificar patrones complejos y proporcionando una evaluación cuantitativa. El presente trabajo consistió en el diseño de dos nuevos modelos originales desarrollados con la modalidad de Deep Learning, el primero capaz de clasificar estructuras de la región torácica y la presencia de cardiomegalia, el segundo permite segmentar arcos costales posteriores de forma autónoma en radiografías de tórax posteroanterior. Los resultados obtenidos demostraron una exactitud del 100\% para el primer modelo en la clasificación de estructuras torácicas, mientras que para la identificación de cardiomegalia la exactitud fue de $99.2 \pm 0.8 \%$. El segundo modelo de segmentación autónoma tiene una exactitud del $93 \pm 29.0 \%$. A partir de estos resultados y con el desarrollo actual de Deep Learning basado en la clasificación y localización consideramos que esta herramienta permitirá en el futuro automatizar algunos procesos que facilitarán la tarea de todos quienes se relacionan al diagnóstico por imágenes.

Palabras clave: Cardiomegalia; Deep learning; Inteligencia artificial; Radiografías de tórax.
\end{abstract}

Abstract

Artificial intelligence algorithms have developed a great advance in image recognition related tasks, being able to identify complex patterns and providing quantitative information. This paper shows the design process of two new Deep Learning models, the first one capable of classifying thoracic structures and the presence of cardiomegaly; the second one allows posterior rib arches autonomous segmentation in posterior-anterior chest $X$-rays. The results showed $100 \%$ accuracy for the thoracic structures classification model, while for the cardiomegaly identification model, the accuracy was $99.2 \pm 0.8 \%$. The second autonomous segmentation model showed $93 \pm 29.0 \%$ accuracy. Based on these results and with the current Deep Learning development, we consider this tool will help automate processes that will facilitate the task of all those who are related to diagnostic imaging.

Keywords: Artificial intelligence; Cardiomegaly; Chest radiographies; Deep learning.

Raschio E, et al. Inteligencia artificial: Desarrollo de algoritmos de clasificación y segmentación en radiografía de tórax Rev Chil Radiol 2021; 27(1): 08-16.

*Correspondencia: Enzo Raschio / enzo.raschio@mayor.cl

Trabajo enviado el 06 de abril de 2020. Aceptado para publicación el 15 de agosto de 2020.

\section{Introducción}

Desde la introducción del termino diagnóstico asistido por computadoras hace más de 60 años por el médico Gwilym S. Lodwick ${ }^{1}$ en un estudio de radiografías de tórax hasta el desarrollo actual de la inteligencia artificial (IA) se ha generado un cambio de paradigma en las formas de procesamiento de la información. La IA ha llegado a superar la capacidad de deducción y observación humana ${ }^{2,3}$, este avance tecnológico ha basado su desarrollo en emular nuestra manera de aprendizaje en múltiples áreas, como el procesamiento de imágenes y reconocimiento de 
características específicas, creando conceptos tales como Red Neuronal Artificial (Artificial Neuronal Network, ANN $)^{4}$, la cual ha sido ampliamente usada en medicina ${ }^{5}$.

En imagen médica han existido importantes avances ${ }^{6,7}$ ya que la cantidad de información, el almacenamiento y la creación de bases de datos con imágenes requieren de análisis que sean rápidos y eficientes que permitan a los médicos tomar las mejores decisiones con respecto a un paciente. En el área de radiodiagnóstico uno de los exámenes más solicitados es la radiografía de tórax ${ }^{8}$, esto debido a que permite una rápida exploración de la región pulmonar y mediastínica. Algunas de las patologías que han sido estudiadas con IA son las patologías pulmonares como la Neumonía $a^{9,10,11,12}$, Tuberculosis 13,14,15 $^{13}$ y otras doce patologías frecuentes ${ }^{16,17,18,19,20,21,22,23}$ de esta zona anatómica, lo cual demuestra la versatilidad multimodal que poseen estas Redes Neuronales Profundas.

El presente trabajo consistió en desarrollar dos modelos propios de software basados en Deep Learning, uno que permite la clasificación de estructuras torácicas y la identificación de cardiomegalia y un segundo modelo capaz de segmentar de manera autónoma los arcos costales posteriores en radiografías de tórax PA.

Con el uso de estos modelos con algoritmos integrados, podríamos tener información relevante sobre la radiografía de tórax tanto en un criterio diagnóstico como es el de insuflación así como en la interpretación de una patología.

\section{Materiales y métodos \\ Bases de datos}

Se utilizaron dos bases de datos públicas ${ }^{24}$, la primera del National Institutes of Health $(\mathrm{NIH})$ que cuenta con 108.948 radiografías frontales de Tórax, la segunda del grupo Shenzen, China, con 662 radiografías de tórax PA. Todos los datos utilizados son de libre uso para este tipo de estudio y se encuentran anonimizados, cumpliendo así con la ley de responsabilidad y portabilidad de la salud (derecho de la privacidad de los datos de las personas). Ambas bases utilizadas están exentas de una revisión por parte un comité de ética de la institución pertinente, al no relacionar los datos de las personas para este fin.

Las bases de datos poseen un informe radiológico, diferenciándose radiografías con y sin patologías. Dentro de esto, la primera base cuenta con un total de 14 patologías presentes en las 100.000 radiografías de tórax de pacientes adultos (84.312 sujetos sanos y 24.636 sujetos con patología positiva), mientras que en la segunda se cuenta con un total de 326 sujetos sanos (especificados con un cero en el informe) y 336 sujetos con tuberculosis positiva (especificados con un uno en el informe), dentro del análisis de estos 662 pacientes, se diferenciaron 641 adultos y 21 pediátricos. Las características demográficas de los sujetos relacionados a las imágenes de estudio se encuentran representadas en la tabla 1.

\section{Criterios de aceptación}

Todas las imágenes obtenidas fueron sometidas a la aplicación del criterio de evaluación de la radiografía de tórax ${ }^{25}$ por parte de los investigadores. Las imágenes utilizadas para el reconocimiento de estructuras (región torácica y arcos costales posteriores) fueron libres de hallazgos patológicos con el fin de optimizar el aprendizaje del modelo. Por otro lado, para el reconocimiento de la patología de cardiomegalia se utilizaron imágenes diagnosticadas con cardiomegalia.

\section{Criterios de exclusión}

Las imágenes con rotación del paciente y que no abarquen desde los ápices pulmonares hasta los recesos costofrénicos ${ }^{25}$ fueron excluidas de este estudio por no cumplir con los criterios de evaluación de este tipo de radiografía. A la vez, se excluyó a todo paciente que presentara las siguientes patologías: Hemotórax, hidrotórax, patrón intersticial y/o alveolar extenso. Esto se debe a que son entidades patológicas con la capacidad de enmascarar en distintas medidas

Tabla 1. Características técnicas y demográficas de las bases de datos.

\begin{tabular}{|c|c|c|c|c|c|c|c|}
\hline $\begin{array}{l}\text { Base de } \\
\text { datos }\end{array}$ & $\begin{array}{l}\text { Pacientes } \\
\text { sanos } \\
\text { (n) }\end{array}$ & $\begin{array}{l}\text { Pacientes } \\
\text { patológicos } \\
\text { (n) }\end{array}$ & $\begin{array}{l}\text { Tipo de } \\
\text { archivo }\end{array}$ & CR/DR ${ }^{*}$ & $\begin{array}{l}\text { Resolución } \\
\text { (píxeles) }\end{array}$ & $\begin{array}{c}\text { Hombres } \\
(\%)\end{array}$ & $\begin{array}{l}\text { Edad } \\
\text { (años) }\end{array}$ \\
\hline Shenzen & 326 & 336 & PNG & DR & $\begin{array}{c}948-3001 x \\
1130-3001\end{array}$ & 66.4 & $33.4 \pm 14.0$ \\
\hline $\mathrm{NIH}^{\star *}$ & 84.312 & 24.636 & PNG & $\mathrm{CR}$ & $\begin{array}{c}1024 x \\
1024\end{array}$ & 48.2 & $59.6 \pm 15.1$ \\
\hline
\end{tabular}


los arcos costales en función de su progresión, lo que resulta negativo para el aprendizaje en la fase de entrenamiento. Sin embargo, sólo en la aplicación del primer modelo para la clasificación de cardiomegalia, fueron usadas imágenes de esta entidad patológica para las 3 fases de procesamiento y desarrollo que posee este modelo de software propio.

Toda imagen radiológica de tórax que no correspondiese a un posicionamiento PA de los pacientes fue descartada del estudio. Esto fue corroborado posterior a la randomización de las imágenes a utilizar, verificando la información de posicionamiento que se encontraba disponible en cada uno de los grupos de imágenes utilizados.

\section{Metodología}

Las radiografías de tórax PA fueron indexadas mediante Photopad ${ }^{26}$ desde su tamaño original, a matrices de 256 × 256 y 512 × 512, y luego convertidas a formato de Portable Network Graphics (PNG) y Tagged Image File Format (TIFF), con el propósito de reducir los tiempos de procesamiento de imágenes con altas matrices en ambos softwares, utilizando el software Photopad $^{26}$. Las imágenes fueron procesadas en un computador con un sistema operativo Windows 10 (Home Single Language) con un marco de trabajo. NET en MATLAB Deep Learning Toolbox ${ }^{27}$ y Python Anaconda ${ }^{28}$, con el driver CUDA v10.1.243 (NVIDIA Corporation, Santa Clara California). El equipo utilizado contiene un procesador i5-7200U de $2.5 \mathrm{GHz}, 1$ TB de disco duro, 16 GB de memoria RAM y un procesador gráfico NVIDIA 940 MX de 2GB.

Los grupos de imágenes utilizados en este estudio fueron seleccionados aleatoriamente mediante la función "randit" en Python 3.7.029 para la posterior aplicación de las fases de entrenamiento, validación y prueba, las cuales comprenden el funcionamiento y la evaluación del rendimiento de cada modelo desarrollado ${ }^{30,31}$ y que se describen a continuación:

- Fase de entrenamiento: Aprendizaje de las características de interés por parte de los softwares (bordes, densidades, tamaño), a partir de imágenes distribuidas en esta fase.

- Fase de validación: Realización de una prueba de lo aprendido mediante la obtención de resultados positivos y negativos para la obtención de los parámetros de rendimiento del modelo

- Fase de prueba: A diferencia de la fase de validación, en esta fase se busca realizar una comparación de resultados con otra entidad del mismo tipo destinada a la misma tarea, o realizar una comparación con las capacidades humanas. Las imágenes fueron pre-procesadas de forma manual previo a la etapa de entrenamiento ${ }^{32}$ de dos distintas maneras para cada uno de los modelos, para el primer modelo (modelo 1) se utilizó una Región de Interés (ROI) en forma de polígono con el software
Image $\mathrm{J}^{33}$ para crear 4 grupos de imágenes, (Figura 1):

1. Imágenes sin pulmones

2. Imágenes sin silueta cardiomediastínica y segmento cérvico-torácico de la columna vertebral

3. Imágenes solo de corazón

4. Imágenes de la caja torácica
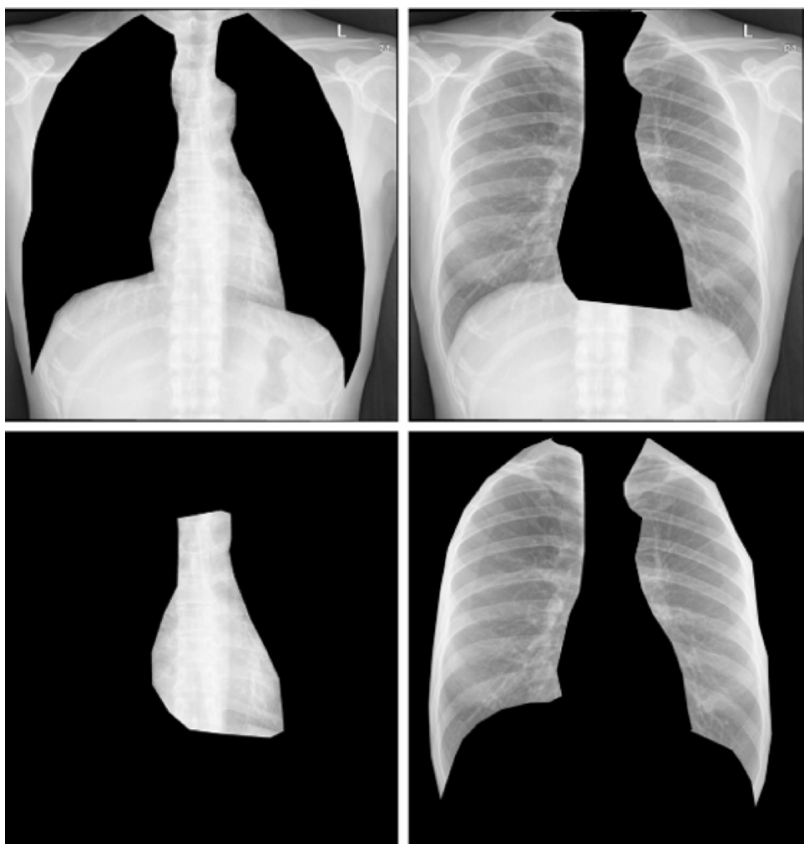

Figura 1: Radiografías de Tórax Postero-Anterior procesadas en los 4 formatos de clasificación. Imagen superior izquierda corresponde al primer grupo, imagen superior derecha al grupo 2, imagen inferior izquierda grupo 3 e imagen inferior derecha grupo 4.

El segundo procesamiento (para el modelo 2) consistió en el etiquetado manual de los arcos costales, mediante el uso del software Labelme ${ }^{34}$ con la herramienta de ROI's con formato de polígono considerando un mínimo de 10 puntos por cada arco costal (Figura 2) esto fue realizado en 1.000 imágenes de sujetos sin patologías diagnosticadas (326 imágenes de la base de datos Shenzen y 674 imágenes de la base de datos del $\mathrm{NIH}$ ).

El modelo 1 es un modelo Bayesiano ${ }^{35,36,37,38}$ pre entrenado, es decir, expuesto previamente a diversas imágenes, las que corresponden a la figura 1. En la figura 3 , se explica gráficamente el funcionamiento de esta red neuronal convolucional. De las imágenes de los 4 sub grupos se realiza una extracción de características de cada uno, con el fin de generar una base de aprendizaje para la posterior clasificación de una de estas estructuras de forma autónoma. 


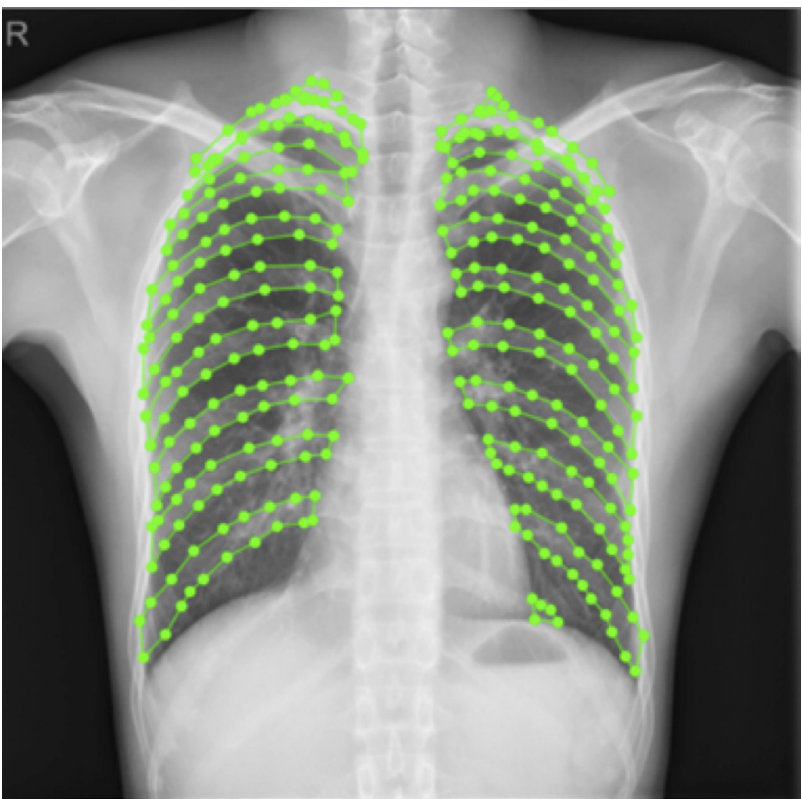

El modelo 2, esquematizado en la figura 5, fue desarrollado utilizando las imágenes procesadas con el etiquetado de arcos costales (Figura 2). Este modelo contempla el uso de 3 capas convolucionales representado en la figura 4 (referente a las aplicaciones de filtros a la matriz de la imagen que ingresa a la red de procesamiento) en la estructura del código.

Para ambos modelos se analizó el parámetro de exactitud, el cual fue obtenido a partir de los resultados de la fase de validación y prueba. Lo anterior acorde al procesamiento de cada modelo, los cuales, categorizaron cada una de las imágenes como exitosa o errónea, en relación a los aspectos aprendidos en la fase de entrenamiento. En base a esto, se realizó para el modelo 1 un análisis contemplando su metodología de clasificación Bayesiana ${ }^{35,36,37,38}$, la cual, contempla la clasificación de resultados como un árbol de decisiones probabilístico de 2 posibilidades en este modelo.

Figura 2: Radiografía de Tórax posteroanterior. Perteneciente al Set del National Institute of Health, con segmentación de arcos costales individuales.

Sets de Entrenamiento y Validación
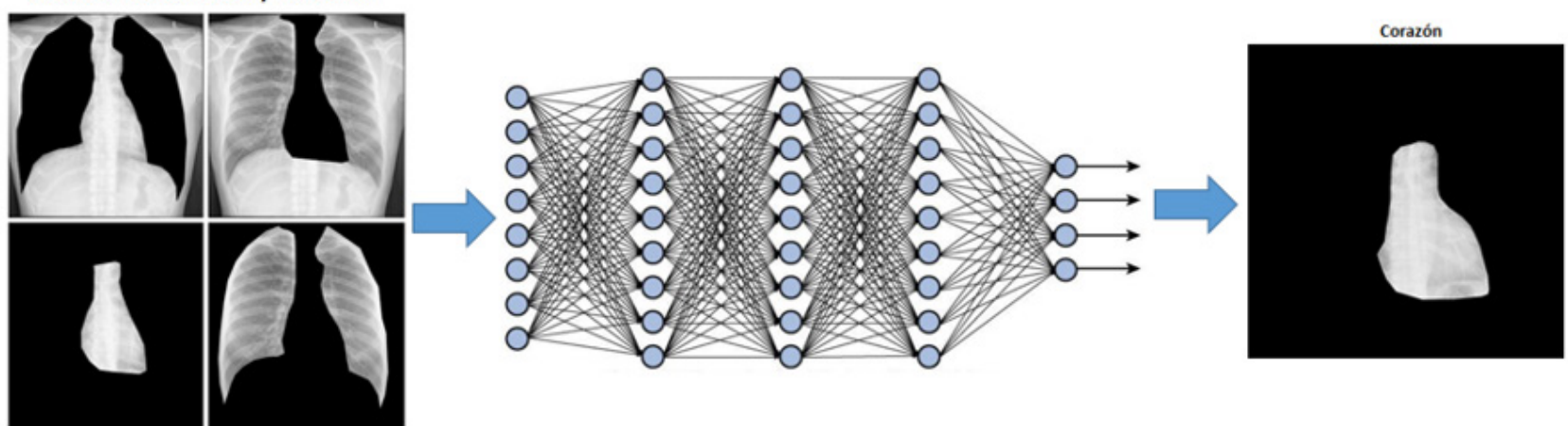

Figura 3: Diagrama de procesamiento de la Red Neuronal de Aprendizaje profundo del modelo 1.

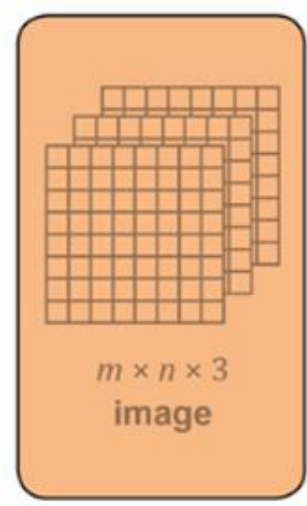

Entrada
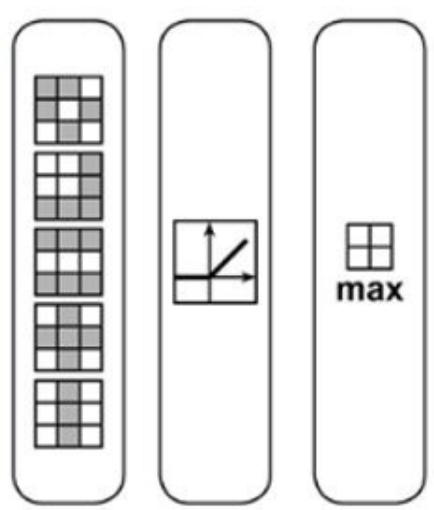

Convolución, Pooling y ReLu

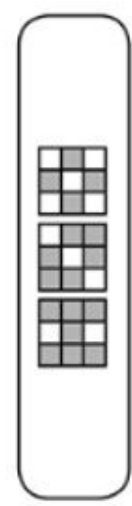

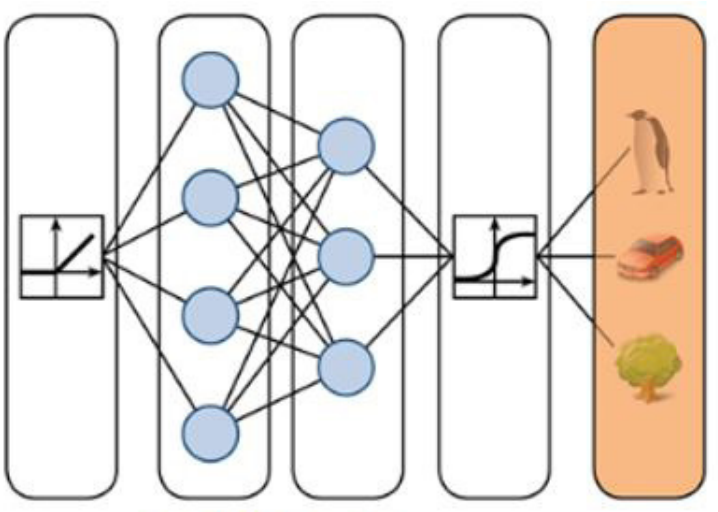

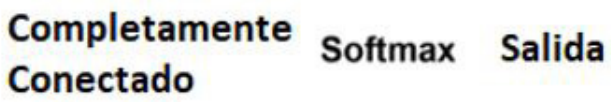

Figura 4: Modelo estructural de clasificación de estructuras y cardiomegalia ${ }^{39}$. 

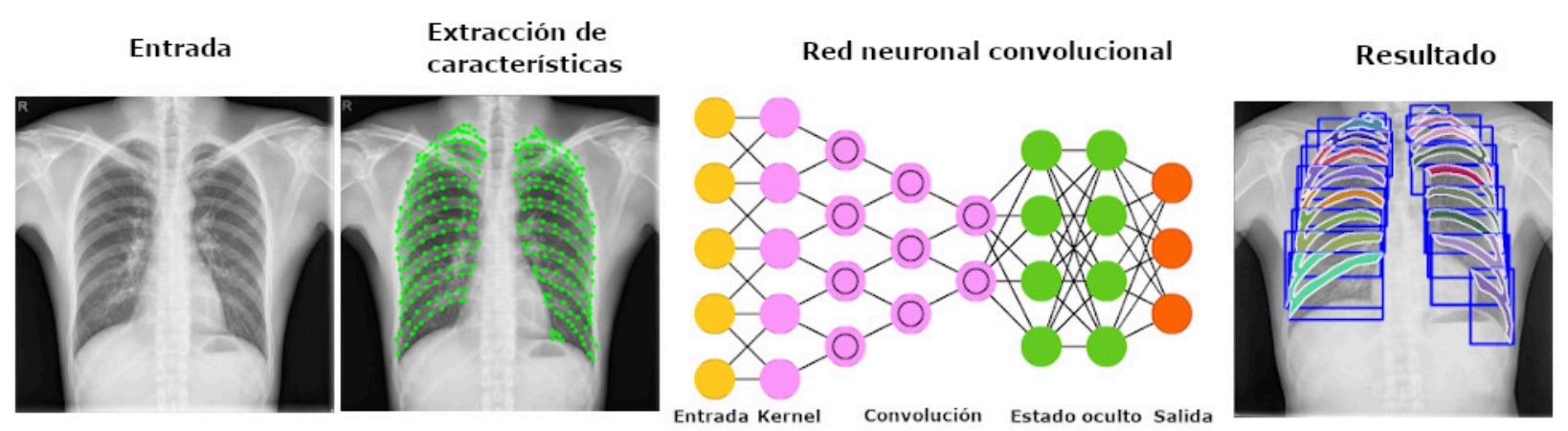

Figura 5: Red de segmentación del modelo 2.

Exclusivamente para el primer modelo se realizó un análisis de curvas de exactitud en el tiempo, las cuales fueron extraídas y generadas a partir de los resultados obtenidos en la fase de entrenamiento y validación. Además, para el modelo 1, fue obtenida la tasa de pérdida de aprendizaje en el tiempo, con el fin de mostrar la variación en la pérdida de información por cada iteración asociada al proceso de aprendizaje del modelo.

Para la evaluación del modelo 2 , se propone en este trabajo una nueva metodología denominada Tasa de Impacto Simulada (TIS), la cual permite una medida evaluativa de la cantidad de arcos costales posteriores segmentados en las imágenes. La TIS se calculó respecto a los datos obtenidos en la fase de prueba versus la observación de los autores (tomando en cuenta los resultados del primer autor como escala de referencia), considerando el conteo de arcos costales posteriores, acorde a la siguiente fórmula:

TIS $=\frac{\begin{array}{l}\text { Radiografías de Tórax PA con menos } \\ \text { de } 10 \text { arcos costales evaluables }\end{array}}{\begin{array}{l}\text { Número Radiografías de Tórax PA } \\ \text { evaluadas por el software }\end{array}} \times 100$

Esta misma fórmula fue aplicada por los autores para obtener el conteo de arcos costales posteriores en el grupo de imágenes de prueba.

\section{Resultados}

En la tabla 2 se encuentran los resultados obtenidos para cada uno de los modelos en función de los respectivos campos de evaluación.

Se obtuvo una exactitud para el primer modelo, tanto en el proceso de validación como en el proceso de prueba de un $100 \%$ al clasificar de manera exitosa las estructuras del mediastino (Figura 5). El proceso de entrenamiento observado en el gráfico 1, contempló un máximo de 100 pasos para el uso de los vectores definidos para el entrenamiento (concepto conocido como Epoch), utilizando sólo una GPU, obtuvo una duración de 1.25 minutos de procesamiento, mientras que para el proceso de validación y prueba se obtuvo un tiempo de 3.7 segundos, considerando un volumen de 250 imágenes randomizadas para este último.

Para el modelo 2 de segmentación autónoma de arcos costales posteriores se obtuvo una exactitud en la fase de validación correspondiente a un $91.0 \pm 20.4 \%$, con un tiempo total de operación en las tres fases de 15 minutos para el procesamiento de todo el set de imágenes, el cual, puede apreciarse en la figura 5. A partir de la aplicación de los datos de la fase

Tabla 2. Resultados de parámetros obtenidos de los softwares diseñados.

\begin{tabular}{|lcc|}
\hline Parámetro & Modelo 1 & Modelo 2 \\
\hline Exactitud en Cardiomegalia (\%) & $99.2 \pm 0.8$ & - \\
Tasa de pérdida Clasificación de sub grupos (\%) & 32 & - \\
Tasa de pérdida Cardiomegalia (\%) & 39 & - \\
Exactitud Fase de Validación (\%) & 100 & $91.0 \pm 29.0$ \\
Batch size & 100 & 100.000 \\
Epochs & 400 y 1000 & 1.000 .000 \\
Exactitud Fase de Prueba (\%) & - & $99.0 \pm 2.6$ \\
TIS*(\%) & - & $9.6 \pm 2.4$ \\
${ }^{*}$ TIS: Tasa de Impacto Simulada & & \\
\hline
\end{tabular}




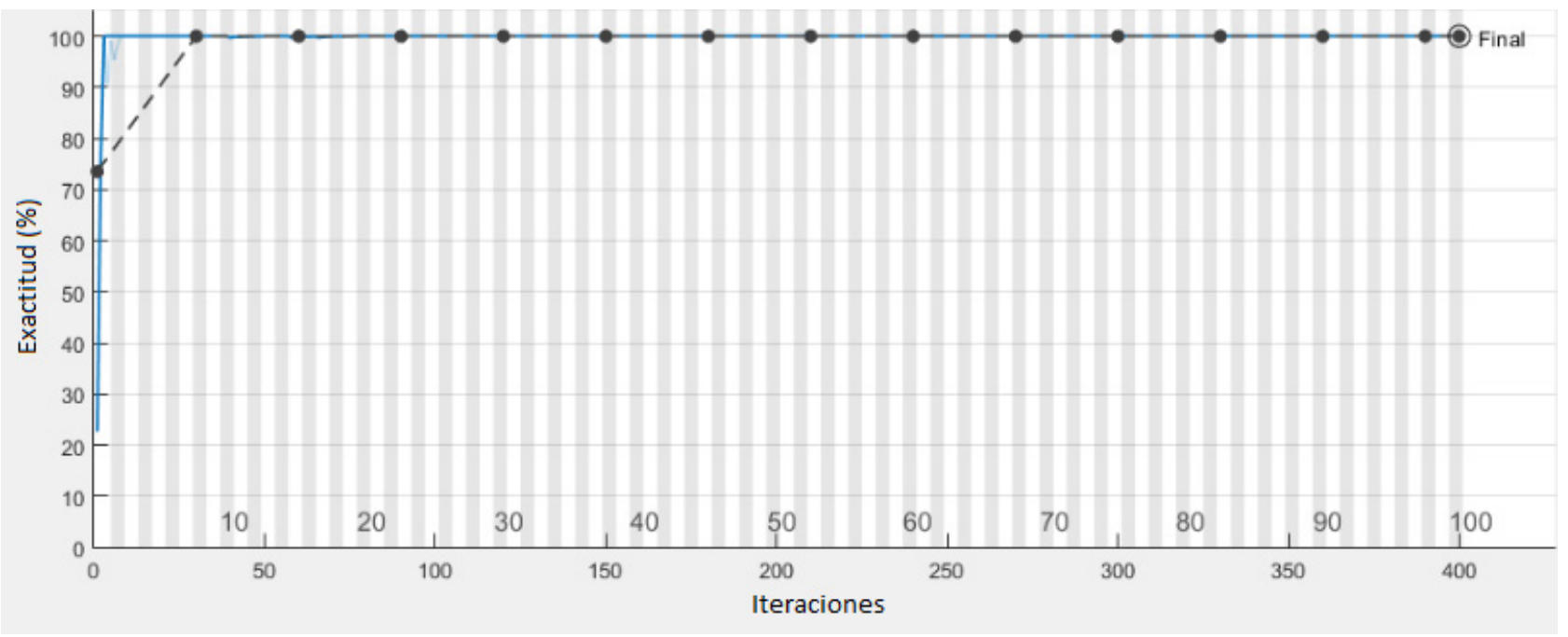

Gráfico 1. Exactitud de la fase entrenamiento y validación del modelo 2 en un periodo de 400 iteraciones.

*El concepto de iteración alude a una medida temporal del procesamiento de las imágenes.

*La línea punteada negra corresponde a la fase de entrenamiento y la línea continua azul a la fase de validación.

de prueba se obtuvo una exactitud de $93.0 \pm 29.0 \%$, con una dispersión asociada a las tres instancias de ejecución de esta fase. Paralelamente la exactitud obtenida para primer autor fue del $100 \%$, mientras para el segundo de $98 \%$ sin dispersión asociada.

Para la obtención de la Tasa de Impacto Simulada, se obtuvo un resultado de $9.6 \pm 2.4 \%$ de radiografías de Tórax PA que no cumplen con el criterio de inspiración aceptado como normal ${ }^{29}$ utilizando el set de prueba.

Los resultados aplicados a la identificación de Cardiomegalia en el modelo 1 (Figura 6) fueron de una exactitud del $100 \%$, asociada a parámetros de entrenamiento, los cuales, fueron de 100 Epochs y 400 iteraciones contempladas para un tiempo de 15 minutos y 14 segundos de análisis de todas las imágenes del set de estudio, abarcando un tiempo de clasificación de 0.41 segundos en la clasificación por imagen. Sin embargo, se obtuvo una variación de la exactitud con la modificación de los vectores de entrenamiento, dando como resultado un $98,4 \%$, en 1 minuto 26 segundos con 10 Epochs y 40 iteraciones efectuadas en la fase de entrenamiento del modelo.

\section{Discusión}

Los resultados obtenidos para el primer modelo clasificatorio de estructuras pertenecientes a la región de tórax y detección de cardiomegalia, se atribuyen a las características de las imágenes utilizadas, principalmente el factor de la matri ${ }^{32}$ y la incorporación de la modalidad de Deep Learning en la estructura del código desarrollado. Esto ha sido avalado por diversos autores tales como Lakhani y Sundaram ${ }^{13} y$ Luo et $\mathrm{al}^{40}$, quienes en sus trabajos emplearon estos
2 elementos, obteniendo un Área Bajo la Curva de 0.90 y 0.918 con el uso de la inteligencia artificial "Alexnet" sin un pre entrenamiento previo (aludiendo al concepto de no haber sido expuesta previamente a imágenes de cualquier tipo).

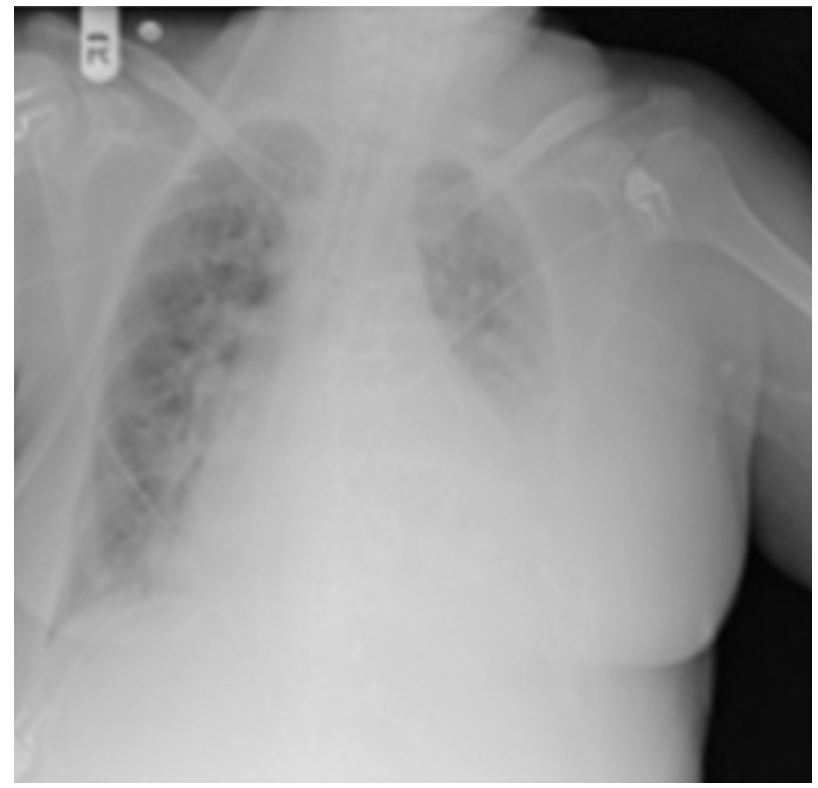

Figura 6: Imagen izquierda radiografía de Tórax PA procesada por el modelo 2. Imagen central perteneciente al grupo de "Corazón" clasificada por el modelo 1. Imagen derecha perteneciente al sub grupo de cardiomegalia clasificada por el modelo 1. 
Por otra parte, la disminución de la Tasa de Pérdida de Exactitud, no resultó un factor determinante en el desempeño de este modelo, debido a que ésta se rectifica o cae a 0 posterior a la cuarta iteración en la fase de entrenamiento, como se puede apreciar en el gráfico 2. Es por esta razón, que la implementación de la estrategia de aumentar la cantidad de iteraciones en el tiempo posee un efecto positivo en el rendimiento de la tasa de aprendizaje de la fase de entrenamiento y validación, aun si esto significar el aumento del tiempo de procesamiento del software.

La identificación de cardiomegalia en el modelo 1 resultó ser exitosa en el proceso de clasificación, al conseguir diferenciar los pacientes con la patología versus de aquellos sin hallazgos patológicos. Esto resulta compatible con los resultados obtenidos por Que et al, donde, CardioXNet ${ }^{41}$ obtuvo una exactitud de $93,75 \%$ en su procedimiento, sin embargo, mediante la utilización del procedimiento de segmentación, lo que implica una arquitectura diferente de la red comparada a nuestro modelo.

En la prueba para la detección de cardiomegalia, se aplicó una estrategia utilizada en algoritmos de tipo bayesiano, en la cual, se realizó una poda

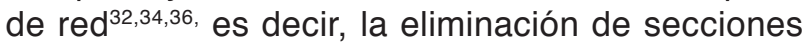
del código con contribuciones mínimas o nulas a la optimización de los resultados. Sin embargo, su aplicación tiene como consecuencia una disminución en la exactitud obtenida, que se evidencia de manera sutil en los resultados obtenidos en la aplicación a la detección de cardiomegalia del mismo modelo, en donde, se ve una baja del $1,6 \%$ en la exactitud.

Por otra parte, resultados obtenidos por el modelo 2 si bien no son iguales o superiores a los obtenidos por el primer y segundo autor de este trabajo, estos resultan alentadores y acorde a los esperados con la aplicación de la modalidad de Deep Learning. Sin embargo, debido al limitado número de imágenes utilizado para la fase de entrenamiento en este trabajo presenta una variación en la exactitud, que debe ser rectificada con el aumento de imágenes. Es por esto que una estrategia compensatoria a este problema fue el aumento de los datos (concepto conocido como "data augmentation") ${ }^{42,43}$, lo cual permitió ampliar el set de entrenamiento de forma digital con la generación de 1.100 imágenes adicionales, lo que en consecuencia tuvo un impacto positivo en el resultado final obtenido. Cabe destacar que las imágenes generadas de esta manera, no tuvieron rotaciones angulares que perjudicaran la imagen obtenida.

Una limitación detectada en el análisis realizado al modelo 2, en la fase de prueba, corresponde a una baja en la segmentación de los arcos costales posteriores de la región inferior de la caja torácica, lo cual, resulta compatible con el estudio realizado por Loog y Van Ginneken ${ }^{44}$ quienes basaron su método de segmentación bajo la metodología de la Clasificación de Pixel Iterada Contextual, obteniendo una exactitud de $0.86 \pm 0.06$ en la prueba de su modelo, concluyendo que las áreas más repetitivas en la falla de la segmentación costal, resulta ser el área inferior correspondiente a la novena y décima estructura costal posterior, por la pérdida de densidad radiológica suficiente para su identificación o por la presencia de estructuras como una trama alveolar prominente (en pacientes sanos), sin embargo, la baja exactitud comparada a nuestro modelo desarrollado puede darse por la menor cantidad de imágenes (25 radiografías de tórax PA) utilizadas para su prueba y/o el uso de Machine Learning, lo cual no da un componente de profundidad al aprendizaje de su modelo.

Las limitaciones pertinentes a este estudio fueron encontradas respecto a la capacidad de procesamiento del ordenador utilizado, el cual presentó un límite respecto a la cantidad de recursos gráficos disponibles para la ejecución del software con una cantidad mayor de imágenes. En base a esto, para el uso de imágenes de una matriz estándar en radiología se propone el uso de un equipo dedicado

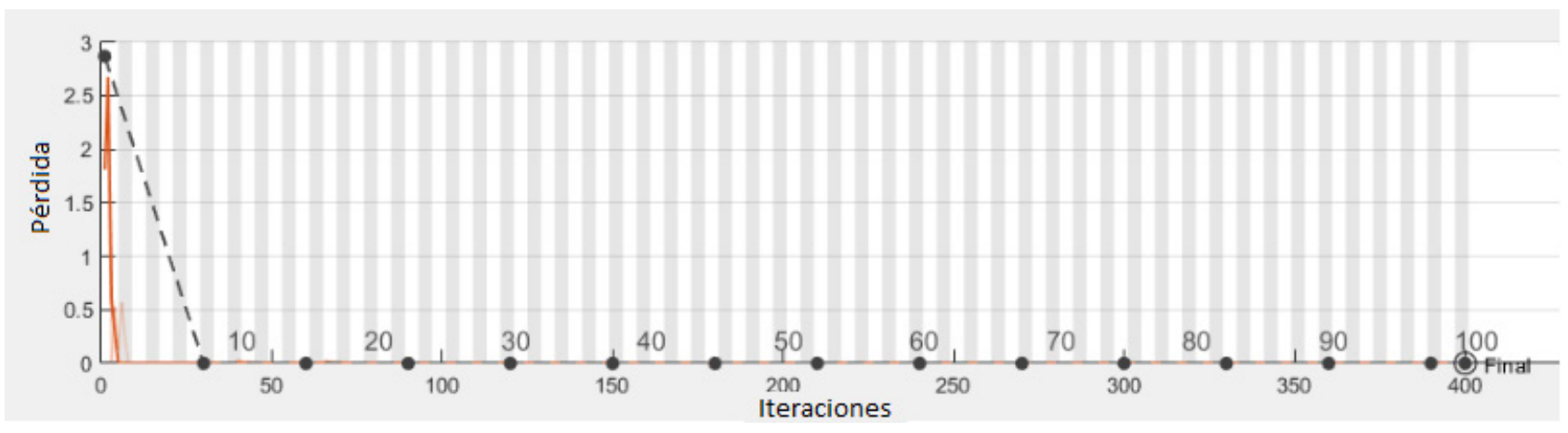

Gráfico 2. Tasa de pérdida de exactitud en la fase de entrenamiento (línea punteada negra) y validación (curva naranja) en el tiempo del software de clasificación modelo 1. 
con la capacidad de procesamiento adecuada para realizar el proceso de indexación de matriz de manera eficiente, tanto en el procesamiento de los atributos de la imagen de entrada, como en el tiempo de realización. Éste será beneficioso en un cálculo más exacto y preciso de la Tasa de Impacto Simulada, debido a que el valor obtenido presenta una variación que momentáneamente no presenta un uso en proceso de calidad.

Se deja abierto en investigaciones a futuro la posibilidad de incluir el uso de radiografías de tórax con presencia de rotación asociada al paciente en estudio, para así poder cuantificar el impacto de este fenómeno, tanto en los resultados de tiempo de procesamiento, como en la exactitud entregada por el segundo modelo respecto al conteo de arcos costales posteriores, ya que estos eventos o condiciones sujetas a la imagen condicionarían al uso de un set de entrenamiento más amplio que el utilizado para el presente estudio. A su vez, se debería considerar el desarrollo de una estructura de código que contemple la posibilidad de solucionar la pérdida de conteo de arcos costales posteriores a nivel del tercio inferior del tórax, específicamente en el área de las cúpulas diafragmáticas, la cual, se considera como un área en donde muchos softwares previos a este tienden a fallar la segmentación de arcos costales por la pérdida de densidad del arco costal, como su mezcla con una densidad similar en las cúpulas diafragmáticas.

En lo referente a la investigación en cardiomegalia, futuros trabajos podrían contemplar no solamente el uso de la clasificación por análisis de pixel de las imágenes de cardiomegalia, sino que deberían visionar el uso de una estrategia mixta, es decir un paradigma de clasificación y segmentación con el fin de aumentar el respaldo de los resultados de clasificación, como a su vez delimitar el área específica de la patología con el uso de Deep Learning.

\section{Conclusión}

De las diversas ramas de la inteligencia artificial aplicada a los problemas de imágenes médicas, se puede concluir que el uso de la modalidad de Deep Learning en nuestros softwares, otorgaron un aumento en la exactitud comparado a softwares basados en Machine Learning, tanto en la clasificación de patologías, como en la segmentación de estructuras de importancia como los arcos costales posteriores, que se encuentran presentes en la evaluación de la calidad en radiografías de tórax PA. Sin embargo, aún se debe mejorar el rendimiento obtenido para el modelo 2, con el fin de lograr un estándar que iguale o supere al observador humano en el conteo de arcos costales posteriores y que asegure la entrega de un resultado óptimo para su uso en instituciones de salud.

\section{Declaración de conflictos de interés}

En este estudio no se presentaron conflictos de interés entre los autores y colaboradores.

\section{Referencias}

1. Lodwick GS. Computer-aided diagnosis in radiology. A research plan. Invest Radiol; 1966; 1: 72-80.

2. Silver D, Huang A, Maddison CJ, Guez A, Sifre L, Van den Driessche G, et al. Mastering the game of go with deep neural networks and tree search. Nature 2016; 529: 484-489.

3. Campbell M, Hoane A, Hsu F. Deep Blue Artif. Intell. Elsevier. 2002; 134: 57-83.

4. Ramesh AN, Kambhampati C, Monson JRT, Drew PJ. Artificial intelligence in medicine. Ann R Coll Surg Engl 2004; 86(5): 334-338.

5. Steimann F. On the use and usefulness of fuzzy sets in medical Al. Artif Intell Med 2001; 21: 131-137.

6. Herweh C, Ringleb PA, Rauch G, Gerry S, Behrens $L$, Mohlenbruch M, et al. Performance of e-ASPECTS software in comparison to that of stroke physicians on assessing CT scans of acute ischemic stroke patients. Int J Stroke 2016; 11: 438-445.

7. Chen L, Bentley P, Rueckert D. Fully automatic acute ischemic lesion segmentation in DWI using convolutional neural networks. Neurolmage Clin. 2017 mayo; 15: 633-643.

8. Prochaska G. IMV Reports General X-ray Procedures Growing at 5.5\% per Year, as Number of Installed X-ray Units Declines. prweb. 2020 (citado 20 marzo 2020). Disponible en: http://www.prweb.com/releases/2011/2/ prweb8127064.htm

9. Rajpurkar P, Irvin J, Zhu K, Yang B, Mehta H, Duan T, et al. CheXNet: Radiologist-Level Pneumonia Detection on Chest X-Rays with Deep Learning. arXiv1711.05225. 2017: 3-9.

10. Singh R, Kalra M, Nitiwarangkul C, Patti J, Homayounieh F, Padole A, et al. Deep learning in chest radiography: Detection of findings and presence of change. PLOS ONE. 2018; 13(10): 1-12.

11. Islam MT, Aowal A, Minhaz AT, Ashraf K, Sep CV. Abnormality Detection and Localization in Chest XRays. arXiv 1705.09850. 2017: 1-16.

12. Zech JR, Badgeley MA, Liu M, Costa AB, Titano JJ, Oermann EK. Variable generalization performance of a deep learning model to detect pneumonia in chest radiographs: A cross sectional study. PLoS Med 2018; 15(11): 1-17.

13. Lakhani P, Sundaram B. Deep Learning at Chest Radiography: Automated Classification of Pulmonary Tuberculosis by Using Convolutional Neural Networks. Radiol 2017; 284(2): 574-582.

14. Harris M, Qi A, Jeagal L, Torabi N, Menzies D, Korobitsyn $A$, et al. A systematic review of the diagnostic accuracy of artificial intelligence-based computer programs to analyze chest $\mathrm{x}$-rays for pulmonary tuberculosis. PLoSONE 2019; 14(9): 1-19.

15. Pande T, Cohen C, Pai M, Khan F. Computer-aided detection of pulmonary tuberculosis on digital chest radiographs: a systematic review. Int J Tuberc Lung Dis 2016; 20(9): 1226-1230.

16. Das N, Topalovic M, Janssens W. Artificial intelligence in diagnosis of obstructive lung desease: current status 
and future potencial. Curr Opin Pulm Med 2017; 24(2): 117-123.

17. Taylor AG, Mielke C, Mongan J. Automated detection of moderate and large pneumothorax on frontal chest X-rays using Deep convolutional neural networks: A retrospective study. PLoS Med 2018; 15(11): 1-15.

18. Yanagawa $M$, et al. Application of deep learning (3-dimensional convolutional neural network) for the prediction of pathological invasiveness in lung adenocarcinoma. Medicine 2019; 98(25): 1-7.

19. Huang G, Zhuang Liu, Van der Maaten L, Weinberger KQ. Densely Connected Convolutional Networks. arXiv:1608.06993. 2018; 1-9.

20. He K, Zhang X, Ren S, Sun J. Deep Residual Learning for Image Recognition. arXiv:1512.03385. 2015; 1-12.

21. Islam MT, Aowal A, Minhaz AT, Ashraf K, Sep C V. Abnormality detection and localization in chest $X$ rays. arXiv 1705.09850. 2017; 1-16.

22. Deng J, Dong W, Socher R, Li J, Li K, Fei L. Imagenet: A large-scale hierarchical image database. IEEE Comput Vis Pattern Recognit. 2009; 1-8.

23. Wang X, Yifan P, Lu L, Lu Z, Bagheri M, Summers M. Chestx-ray8: Hospital-scale chest x-ray database and benchmarks on weakly-supervised classi_cation and localization of common thorax diseases. IEEE. arXiv:1705.02315. 2017; 1-19.

24. Jaeger S, Candemir S, Antani S, Wáng YX, Lu PX, Thoma G. Two public chest X-ray datasets for computer-aided screening of pulmonary diseases. Quant Imaging Med Surg. 2014; 4(6): 475-477.

25. Frank E, Long B, Smith B. Vísceras Torácicas. Merrill Atlas de Posiciones Radiográficas y Procedimientos Radiológicos. 11va ed. Madrid. Elsevier Mosby. 2010; 514-556.

26. Photopad. Canberra, Australia. NCH Software. 2019. Disponible en: https://www.nchsoftware.com/ photoeditor/es/index.html

27. Deep Learning Toolbox. The Mathworks, Inc. 2019. Disponible en: https://es.mathworks.com/products/ deep-learning.html

28. Wang P, Pierce A, Reeves J, Davis-Felner K, Malkiewicz N, Grant M, et al. Austin, Texas. Anaconda Inc; 2019. Disponible en: https://www.anaconda. com/distribution/\#download-section.

29. Python 3.7.0, Python.org.Inc. (Actualizado 26 octubre 2018). Disponible en: https://www.python.org/ downloads/

30. Zhang J, Harman M, Ma L, Liu Y. Machine Learning
Testing: Survey Landscapes and Horizons. arXiv: 1906.10742. 2019.

31. Xie X, Ho J, Murphy C, Kaiser G, Xu B, Chen T. Testing and validating machine learning classifiers by metamorphic testing. Elsevier 2010; 84(4): 544-558.

32. Islam J, Zhang Y. Towards robust lung segmentation in chest radiographs with deep learning. 2018. arXiv: 1811.12638

33. National Institutes of Health. ImageJ. 2019. Disponible en: http://imagej.nih.gov/ij/download.html

34. Ketaro W. Labelme: Image Polygonal Annotation with Python. 2016. Disponible en: https://github. com/wkentaro/labelme

35. Hinton G.E, Van Camp D. Keeping the neural networks simple by minimizing the description length of the weights. In Proceedings of the Conf Comput (ACM). 1993; 5-13.

36. Louizos C, Ulrich K, Max Welling. Bayesian compression for deep learning. NIPS'17, 2017.

37. Guo Y, Yao A, Chen Y. Dynamic network surgery for efficient dnns. In advances in neural information processing systems. NIPS'16. arXiv: 1608.04493. 2016; 1379-1387.

38. Han S, Mao H, Dally W.J. Deep compression: Compressing deep neural networks with pruning, trained quantization and huffman coding. ICLR. arXiv: 1510.00149. 2016; 1-14.

39. Matlab for Engineers. Emiratos Árabes. (Citado el 15 de mayo del 2020). Disponible en: http://matlab4engineers.com/lesson/cnn-architecture/?lang=e $n \& v=5 b c 574 a 47246$

40. Exhibit S, Luo JW, Chong JJR, Applications-general C, Applications- C. Newer Is Better? Comparing deep learning architectures for cardiomegaly detection on chest radiographs. Eur Radiol 2019; 1-9.

41. Que Q, Tang Z, Wang R, Zeng Z, Wang J, Chua M, et al. CardioXNet: Automated detection for cardiomegaly based on deep learning. Conf Proc IEEE Eng Med Biol Soc 2018; 612-615.

42. Perez L, Wang J. The Effectiveness of data augmentation in image classificaion using deep learning. arXiv:1712.04621. 2017; 1-8.

43. Taylor LI Netschke G. Improving deep learning using Generic Data Augmentation. arXiv:1708.06020. 2017; 1-6.

44. Loog M, Ginneken B. Segmentation of the posterior ribs in chest radiographs using iterated contextual pixel classification. IEEE Trans Med Imaging 2006; 25(5): 602-611. 